\title{
National Center for In-situ Tomographic Ultramicroscopy at New York Structural Biology Center
}

Misha Kopylov ${ }^{1}$, Daija Bobe ${ }^{1}$, Ashleigh Raczkowski ${ }^{2}$, Elina Kopylov ${ }^{1}$, Charlie Dubbeldam ${ }^{1}$, Alex Noble $^{1}$, Bridget Carragher ${ }^{1}$ and Clint Potter $^{1}$

${ }^{1}$ New York Structural Biology Center, New York, New York, United States, ${ }^{2}$ NYSBC, New York, New York, United States

The National Center for In-situ Tomographic Ultramicroscopy (NCITU) is one of the four Cryo-Electron Tomography (CryoET) service centers that are part of NIH Common Fund's Transformative High Resolution Cryo-Electron Microscopy (CryoEM) Program and is funded under NIH Grant U24GM139171. We are housed within the New York Structural Biology Center as a part of Simons Electron Microscopy Center (SEMC). NCITU is one of three "spoke" centers with a focus on sample preparation and production of thin specimens suitable for Cryo-Electron Tomography (CryoET). Tomographic data acquisition will be performed by Midwest Center for Cryo-Electron Tomography (MCCET), the central "hub."

Every in-situ workflow is as unique as the biology in question; however, a number of steps is common among all of them. Our "standard" workflow starts at sample preparation and ends with a high-resolution CryoET data collection. NCITU will provide facilities for cell growth, target protein expression and live cell screening. Depending on the needs of a project, cells will be applied to EM grids in bulk or accurately positioned using a micropatterning device. To carefully preserve the state of the biological system, cells will be vitrified by plunge-freezing or high-pressure freezing. They then can be screened on a confocal microscope equipped with a cryo-stage. In a penultimate step, FIB-SEM is used to thin out areas of interest (make lamellas) in the cells to prepare for tomography. Before shipping to the hub for data collection, grids will be screened on-site to ensure the presence of intact, electron-transparent lamellas.

As in-situ methods are rapidly developing, staying at the forefront of technology is one of our main goals at NCITU. One of the first specific aims is to continue development and automation of the "waffle" method of CryoET sample preparation. We will constantly work together with other centers to exchange technological developments, protocols as well as tips and tricks. Established and new technologies will be made available through the cross-training of the users at all skill levels.

Application to the CryoET network will be centrally administered through the "hub" at MCCET. Once application is completed, it will be reviewed for completeness and sent to the user review committee for peer review. In the result, a score will be assigned to the application which will then be added to the queue. Depending on the specific needs of the project - unique equipment, specific methodology or expertiseit will be assigned to one of the specific centers such as NCITU. At this step, during the follow up meeting with NCITU team, we can discuss the details of the project, cross-training program and allocate time.

NCITU is dedicated to developing and providing access to both standard and advanced methods for insitu specimen preparation, and to disseminating this expertise through a CryoET cross-training program designed to serve a wide variety of skill levels and career goals.

References 
Waffle method: A general and flexible approach for FIB-milling small and anisotropically oriented samples

Kotaro Kelley, Pattana Jaroenlak, Ashleigh M. Raczkowski, Edward

T. Eng, Gira Bhabha, Clinton S. Potter, Bridget Carragher, Alex

J. Noble; bioRxiv 2020.10.28.359372; doi: https://doi.org/10.1101/2020.10.28.359372 